\title{
E-Learning System Utilizing Learners' Characteristics Recognized Through Learning Processes with Open Simulator
}

\section{Overcoming Week Points through Dialogs with Avatars in Open Simulator in Learning Processes}

\author{
Kohei Arai \\ Graduate School of Science and Engineering \\ Saga University \\ Saga City, Japan
}

\author{
Anik Nur Handayani \\ Graduate School of Science and Engineering \\ Saga University \\ Saga City, Japan
}

\begin{abstract}
E-learning system utilizing learners' characteristics which is recognized through learning processes with Open Simulator for overcoming week points is proposed. Through dialogs with avatars in the Open Simulator, it is possible to understand learners' week points. Using learners' characteristics, most appropriate subjects and achievement tests are provided by the proposed e-learning system. Experimental results show that the proposed e-learning system is much effective than the conventional e-learning system without utilizing learners' characteristics.
\end{abstract}

Keywords-e-learning system; Q/A System; Open Simulator; learners' characteristics;

\section{INTRODUCTION}

Studies over the years are shown that students had actively and more interactively involved in a classroom discussion or self studies increases by as much as $20 \%$. Meanwhile, IT technology has been seen a tool to encourage student involvement. Today, a large number of portable game consoles and network devices could be representative. Also the blending of those devices is getting popular. It illustrates familiar scenario for today learners.

Virtual worlds such as OpenSimulator require a level of meta-cognition in relation to functioning within them, the translation of movement and actions via an interface medium make the learning such as real learning.

This proposed e-learning system will assist the students in the learning process through the $\mathrm{Q} / \mathrm{A}$ system utilizing avatar using OpenSim as Virtual Education opportunities. There are some related research works. The paper discusses a case study which aims to make research projects more engaging for middle school students by incorporating a variation to the traditional gallery walk workflow [1].

They collect research posters and transfer the media into the shared a virtual world. Present collaborative cheerleading method among virtual world users and sensory effect is using wearable hap tic wrist guard for providing more interactive and immersive [2].
Describing the use of OpenSimulator as a platform for enhanced learning concepts incorporate a variety of desirable attributes [3].

The following section describes the proposed Q/A system utilizing e-learning system with OpenSimulator of avatar followed by some experiments. Then conclusion is described with some discussions.

\section{PROPOSED Q/A SySTEM UTILIZING E-LEARNING SYSTEM WITH OPENSIM OF AVATAR}

\section{A. Problem Statements}

There are some knowhow,

a) How to developing and connecting user avatar and avatar system by using OpenSimulator

b) How to developing Q/A in the system

c) How to connecting avatar system with the knowledge base (i.e. Wikipedia Online)

d) How to parsing the question

e) How to gain the Q/A pair to the knowledge database Namely, grid,

a) Starting with installing, setup user, setup region and learning

b) Developing Q/A system for collaborative virtual

c) By using LSL HTTP request using Linden Scripting Language (LSL) and Wikipedia API

(Application Protocol Interface) using LSL is used for creating interactive content in Second Life.

(4)To get keyword

(5)Gain the Q/A activity to the knowledge database (external database)

OpenSimulator is an open source server platform for hosting virtual worlds. It is compatible with the client 
for Second Life and can host alternative worlds with differing feature sets with multiple protocols. Open Simulator has the following features,

1) Supports online, multi-user $3 D$ environments as small as 1 simulator or as large as thousands of simulators.

2) Supports $3 D$ virtual spaces of variable size within one single instance.

3) Supports multiple clients and protocols - access the same world at the same time via multiple protocols.

4) Supports clients that create $3 D$ content in real time.

5) Supports in world scripting using a number of different languages, including LSLJOSSL, C\# and VB.NET.

where LSL = The Linden Scripting Language (LSL) is used for creating interactive content in Second Life.

Only the single open simulator is used for the proposed Q/A system utilizing e-learning system with OpenSim of avatar.

\section{B. Q/A System}

Referring to the Question Answering Systems: A survey $\rightarrow$ Ali Mohamed Nabil Alam, Mohamed Hassan Haggag (IJRRIS Sept. 2012), the following Q/A system is created. Process flow of $\mathrm{Q} / \mathrm{A}$ is shown in Figure 1. Q/A system consists Question processing, Information processing, and answer processing as follows,

\section{Question Processing}

1) Question Analysis: Identifying the focus can be done using pattern matching (pengenalan pola) rules, based on the question type classification

2) Question Classification: The question is classified by its type: what, who, when, where, why and how.

3) Question Reformulation: The process of extracting keywords such as stop word lists

\section{Information Processing}

\section{1) Information Retrieval:}

Information retrieval systems are usually evaluated based on two metrics - precision and recall. Precision refers to the ratio of relevant documents returned to the total number of documents returned. Recall refers to the number of relevant documents returned out of the total number of relevant documents available in the document collection being searched.

2) Paragraph Filtering: the most relevant documents should contain the question keywords

3) Paragraph Ordering:

Same word sequence score: the number of words from the question that are recognized in the same sequence within the current paragraph window.

\section{Answer Processing}

1) Answer identification: The recognition of the answer type returned by the parser creates a candidate answer

2) Answer extraction: The parser enables the recognition of the answer candidates in the paragraphs. So, once an answer candidate has been identified.

\section{3) Answer validation:}

Confidence in the correctness of an answer can be increased in a number of ways.

We use specific knowledge sources to check answers.

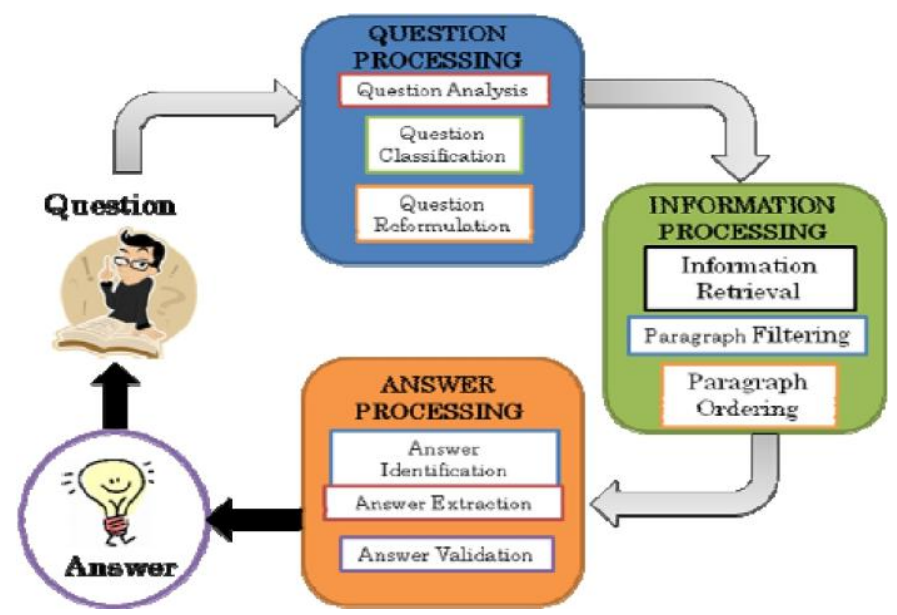

Fig. 1. Process flow of the proposed Q/A system

\section{System Block Diagram}

Figure 2 shows system block diagram of the proposed system. The proposed system is based on Client Server model. There are two avatar systems, one is in the server and the other one is in the client. Both avatars can communicate each other as well as in between client user avatars. Thus the question raised from one user can be shared with all users. The server side avatar is always listening to the question and answer among users using LSL. HTTP request using LSL and PHP for keyword parsing and querying search can be done together with JSON/XML output respond. All the questions and answers are archived in the knowledge base system. OpenSimulator 0.7 .2 creates the basic avatar clothing/body parts set (a ruth) for each avatar so they should be editable separately by each user.

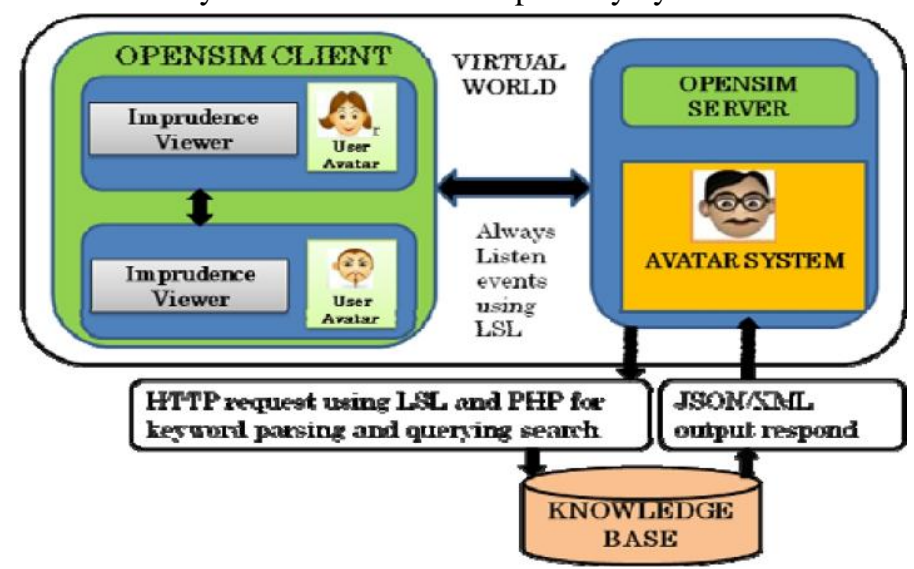

Fig. 2. System block diagram of the proposed system. 
Figure 3 shows system architecture.

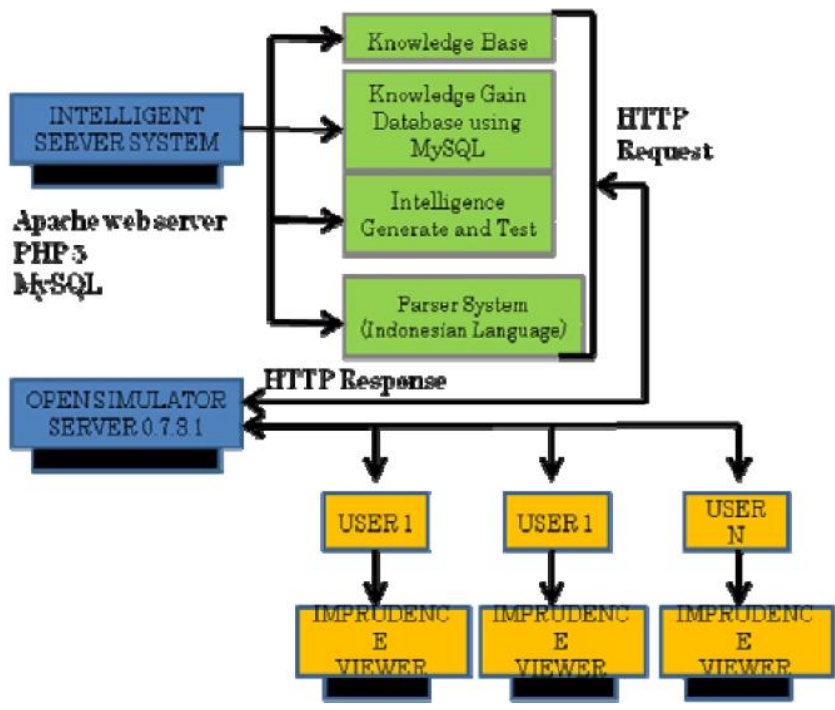

Fig. 3. Proposed system architecture

In the system architecture level, internet interface can be done with Apache web server with web design by PHP 5 . Knowledge base system is based on MySQL. Also, Knowledge base is realized by using Wikipedia API (Application Protocol Interface). Results send in xml format, $\mathrm{xml}$ convert to readable format using PHP. Internet/Local Communication using HTTP Protocol (HTML Post Method) with the following procedure,

HTML Post Method $\rightarrow$

Get $\rightarrow$ is basically for getting data

Post $\rightarrow$ storing updating data or ordering product

\section{Proposed Learning Procedure}

Avatar in the server side has huge knowledge base which contains questions and correct answers through the previous learning processes by users. Avatar in the server side, also can present most appropriate answer which is extracted from the database to the question which is raised from users. These processes are archived every time user makes a question as knowledge.

Furthermore, server side avatar has the capability for characterization of users. In other word, knowledge base is created and updated by user by user. Users learn subjects in a collaborative manner. They can make a question and also can respond to it. These answers are qualified by server side avatar through Wikipedia API. Thus user answers and Wikipedia answers are evaluated and most appropriate answer is provided to the user who makes the question. These correct and most appropriate answers are archived in the database in the knowledge base system.

Specific user makes specific mistakes. Such this fact is a user's character. Server side avatar knows user's character through characterization from the aforementioned learning processes. Thus server side avatar makes corrections when user makes mistakes. Also server side avatar knows users' difficulties. Therefore, avatar makes a plenty of questions for the difficult subjects for the specific users.

\section{IMPLEMENTATION AND EXPERIMENTS}

\section{A. Implimentation}

Open Simulator 0.7.2 can be installed easily. Also PHP, Apache, MySQL are installed easily together with LSL and Wikipedia API. Knowledge base contains questions and answers as well as evaluation results of quality of answers which are evaluated using LSL and Wikipedia API. The knowledge base is composed with tables included in MySQL of database as shown in Figure 4. Figure 4 (a) shows example of questions and answers retrieved result while Figure 4 (b) shows example of retrieved result of correct answers. Figure 4 (c) shows example of the history of achievement test results. It can be done by student by student, by subject by subject, by stage by stage, etc. The table contains field, type, null key default, and extra for the data of knowledge, question, and answer. Using MySQL command, all the questions and correct answers are retrieved. Also it can be referred the history of achievement test results by subject by subject.

\section{B. Avatar Design}

Figure 5 shows examples of avatar designed for the proposed system specifically.

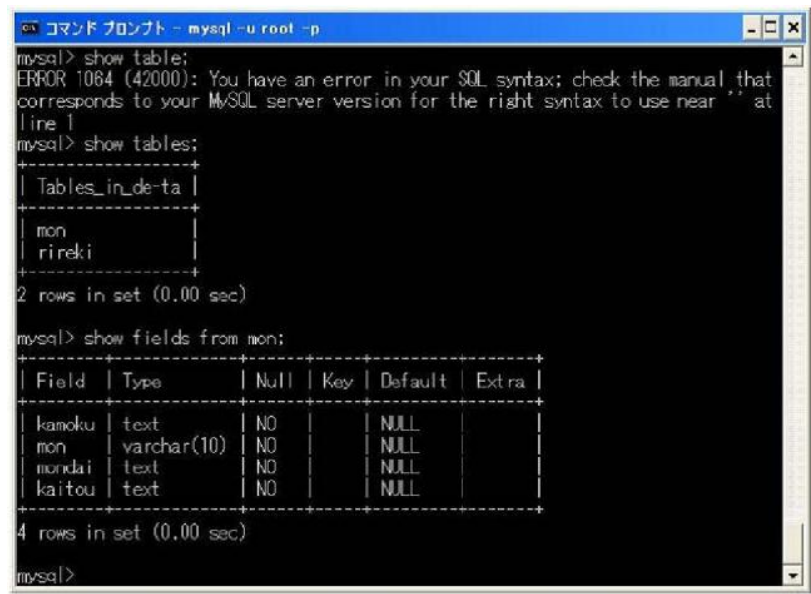

(a)Database content by question and answer

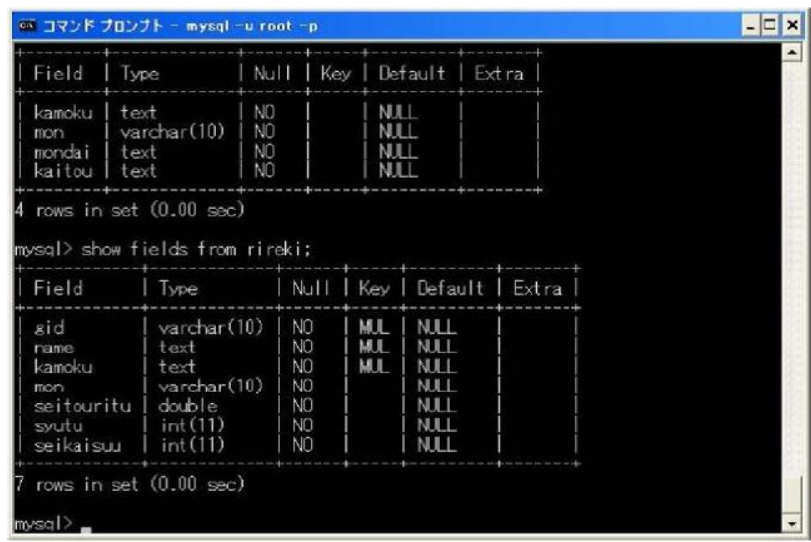

(b)Correct answer retrieval 


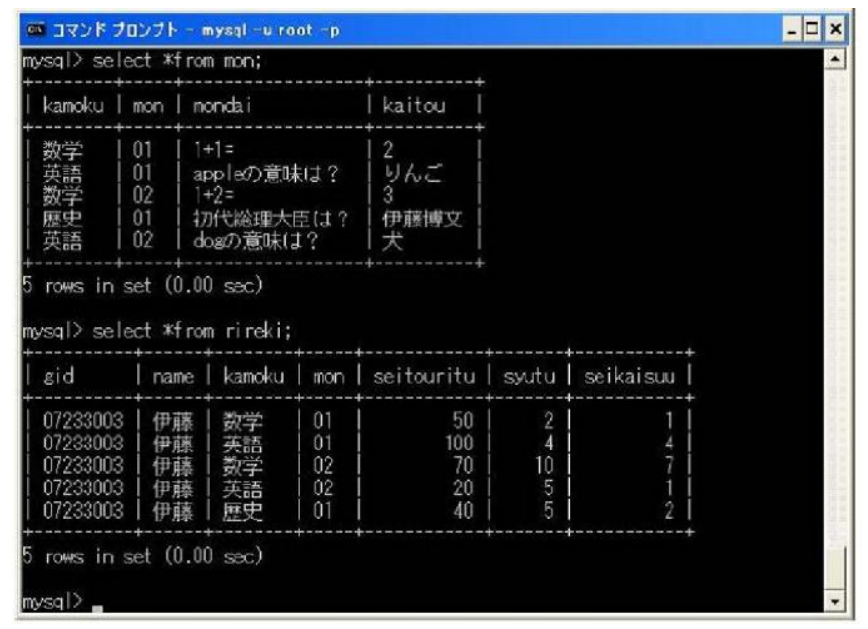

(c)Achievement test result history by subject by

Fig. 4. subject Figure 4 Database contents

It can be done with the following procedure,

1) Click the Inventory Button

2) Create -> New Clothes -> Shirt, Pants, etc

3) Create -> New Body Parts -> Hair, Shape, etc

4) Edit those from the inventory

5) Wear them

An update: SecondLife: SL has since removed the default "Ruth" avatar and replaced it with a set of pre-built custom avatars. If there is ever an issue with your current avatar under either OpenSimulator or SL, your avatar is displayed as a particle cloud effect. OpenSimulator maintains a default avatar very similar to what "Ruth" looked like, however, for additional custom avatars, one would need to build a repository of avatar clothes and body parts (in their server's database, available to all, or in that particular user's inventory alone).

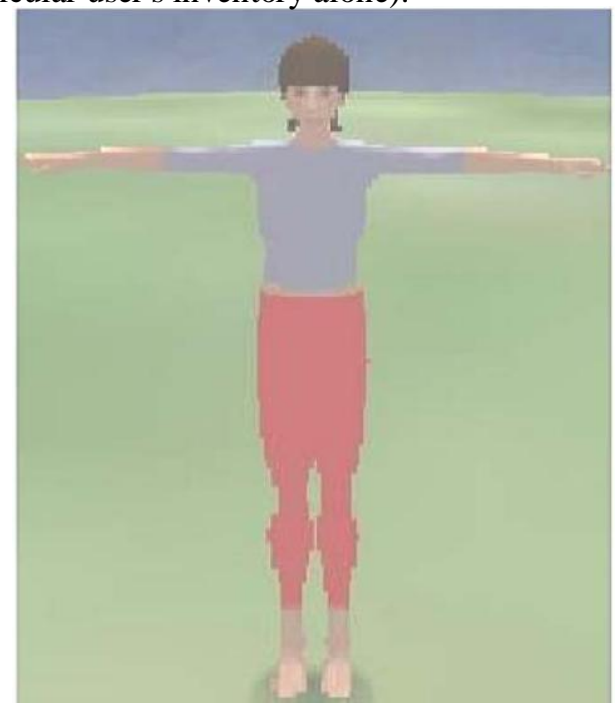

(a)Designed fundamental avatar

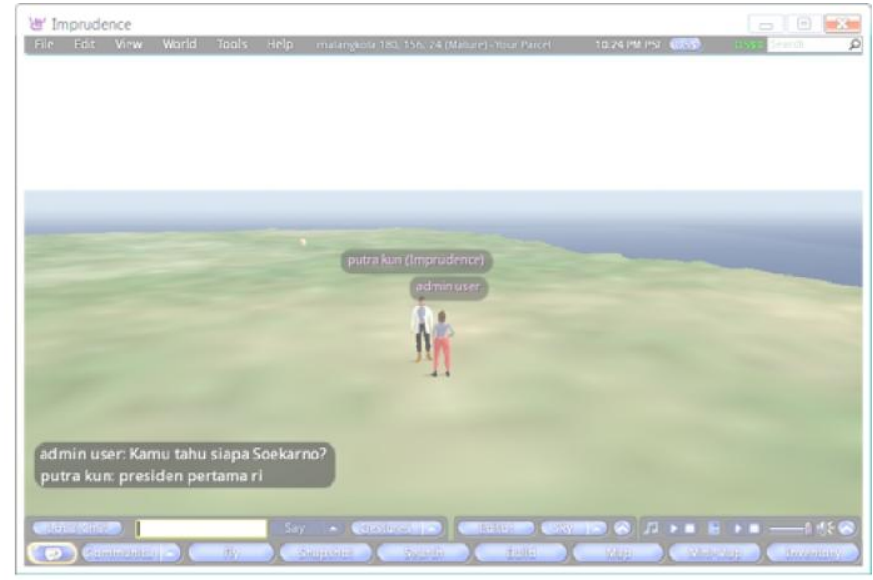

(b)Client user avatars

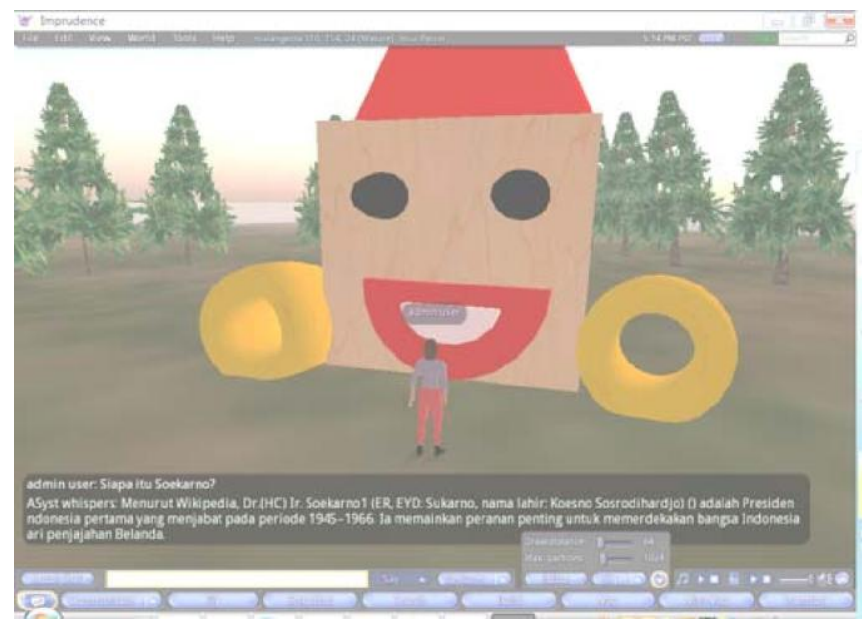

(c)Server avatar

Fig. 5. Designed avatars.

\section{Implementation and Experimental Result}

Q/A system implementation result are shown in Table 1. Q/A system are completed without any trouble.

Experiments of learning performance test with and without avatar are conducted. Three subjects, History, Mathematics, and English reading are prepared. The effectiveness of avatar can be evaluated with achievement test by ten of undergraduate students of Saga University. In accordance with the experimental results, approximately $10 \%$ of improvement is observed for e-learning system with avatar in comparison to that without avatar. The improvements are different by subject by subject. The avatar is effective for the subject of history followed by English reading and mathematics. Although concrete answer can be easily prepared for history and English reading, it is rather difficult to prepare concrete answer for the mathematics subject 
TABLE I. Result from Q/A system installation

\begin{tabular}{|c|c|c|c|}
\hline $\begin{array}{c}\text { QA } \\
\text { Components }\end{array}$ & $\begin{array}{c}\text { Question } \\
\text { Processing } \\
\text { Analysis }\end{array}$ & $\begin{array}{c}\text { Document } \\
\text { Processing }\end{array}$ & $\begin{array}{c}\text { Answer } \\
\text { Processing }\end{array}$ \\
\hline $\begin{array}{c}\text { Question } \\
\text { Classification }\end{array}$ & $\sqrt{ }$ & & \\
\hline $\begin{array}{c}\text { Question } \\
\text { Reformulation } \\
\text { Retrieval }\end{array}$ & $\sqrt{ }$ & $\sqrt{ }$ & \\
\hline $\begin{array}{c}\text { Paragraph } \\
\text { Filtering }\end{array}$ & & $\sqrt{ }$ & \\
\hline $\begin{array}{c}\text { Paragraph } \\
\text { Ordering }\end{array}$ & & $\sqrt{ }$ \\
\hline $\begin{array}{c}\text { Answer } \\
\text { Identification }\end{array}$ & & & $\sqrt{ }$ \\
\hline $\begin{array}{c}\text { Answer } \\
\text { Extraction }\end{array}$ & & & \\
\hline $\begin{array}{c}\text { Answer } \\
\text { Validation }\end{array}$ & & & \\
\hline
\end{tabular}

IV. Conclusions

E-learning system utilizing learners' characteristics which is recognized through learning processes with Open Simulator for overcoming week points is proposed. Through dialogs with avatars in the Open Simulator, it is possible to understand learners' week points. Using learners' characteristics, most appropriate subjects and achievement tests are provided by the proposed e-learning system. Experimental results show that the proposed e-learning system is much effective than the conventional e-learning system without utilizing learners' characteristics.
As the results, the following conclusions are obtained,

1) In this proposed research we focus on collaborative question answer activities in the networked virtual world environment.

2) With the avatar system that connects to the knowledge database, could assist student in providing answers.

3) QA based collaborative learning could be done much attractively than the conventional QA system through Avatars in virtual learning.

\section{ACKNOWLEDGMENT}

The authors would like to thank Mr. Kazauma Itoh of Saga University and the students of State University of Malang, Indonesia who contributed to the experiments.

\section{REFERENCES}

[1] Joshua Schendel, Chang Liu, David Chelberg, Teresa Franklin. 2008. Virtual Gallery Walk, an Innovative Outlet for Sharing Student Research Work in K-12 Classrooms. $38^{\text {th }}$ Frontiers in Education Conference.

[2] Changhyeon Lee, Hye, Min Choi, Yong-Moo Kwon. 2010. Networked Collaborative Group Cheerlading Technology. International Symposium on Ubiquitous Virtual Reality.

[3] Walter Ridgewell, Vive Kumar, Oscar Lin. 2011. OpenSim Virtual World as Platform for Enhanced Learning Concepts. 11 ${ }^{\text {th }}$ IEEE International Conference on Advanced Learning Technologies.

\section{AUTHORS PROFILE}

Kohei Arai, He received BS, MS and PhD degrees in 1972, 1974 and 1982, respectively. He was with The Institute for Industrial Science and Technology of the University of Tokyo from April 1974 to December 1978 also was with National Space Development Agency of Japan from January, 1979 to March, 1990. During from 1985 to 1987, he was with Canada Centre for Remote Sensing as a Post Doctoral Fellow of National Science and Engineering Research Council of Canada. He moved to Saga University as a Professor in Department of Information Science on April 1990. He was a councilor for the Aeronautics and Space related to the Technology Committee of the Ministry of Science and Technology during from 1998 to 2000. He was a councilor of Saga University for 2002 and 2003. He also was an executive councilor for the Remote Sensing Society of Japan for 2003 to 2005. He is an Adjunct Professor of University of Arizona, USA since 1998. He also is Vice Chairman of the Commission "A" of ICSU/COSPAR since 2008. He wrote 30 books and published 322 journal papers 